\author{
Journal of the Operations Research \\ Society of Japan \\ Vol. 37, No. 1, March 1994
}

\title{
RELATIONSHIP BETWEEN QUEUE-LENGTH AND WAITING TIME DISRIBUTIONS IN A PRIORITY QUEUE WITH BATCH ARRIVALS
}

\author{
Yoshitaka Takahashi Masakiyo Miyazawa \\ NTT Laboratories Science University of Tokyo
}

(Received July 9, 1992; Revised March 11, 1993)

\begin{abstract}
We consider a single-server priority queue with batch arrivals. We treat the head-of-the-line (HL) or preemptive-resume (PR) priority rule. Assuming that the arrival process of batches is renewal for each priority class and using the point process approach, we express the individual class queue-length distribution in terms of the waiting time and the completion time distributions. Assuming further a batch Poisson arrival for each class, together with the previous result on the Laplace-Stieltjes transforms for the waiting time and completion time distributions, we derive the $\mathrm{z}$-transform for the queue-length distribution in closed form.
\end{abstract}

\section{Introduction}

We consider a multi-class single-server priority queue with batch arrivals, where the batch interarrival times and the customer service times are respectively independent and identically distributed (i.i.d.) for an individual class. We assume the head-of-the-line (HL) or preemptive-resume (PR) priority rule. We will denote this priority queue by $\overrightarrow{G I^{X}} / \overrightarrow{G I / 1}$ (HL or PR); see Section 3 for its detail description. The purpose of this paper is to obtain the individual class queue-length distribution in terms of the waiting time and completion time distributions. Especially for the multi-class batch Poisson arrival $\overrightarrow{M^{X}} / \overrightarrow{G I / 1}$ (HL or $\mathrm{PR}$ ) priority queue, we will obtain the z-transform for the individual class queue-length distribution in closed form.

So far, there have been few results on the individual class queue-length distribution for the multi-class batch Poisson arrival priority queue. Indeed, for the two-class $\overrightarrow{M^{X}} / \overrightarrow{G I / 1}$ (HL) priority queue, only Hawkes [8] has obtained the z-transform for the queue-length distribution using the supplementary variable approach. It seems difficult to extend this two-class result [8] to the multi-class priority queue, since we have to solve a multi-dimensional functional equation. On the other hand, there have been fruitful results on the Laplace-Stieltjes transforms (LSTs) for the individual waiting time and completion time distributions. For the multi-class $\overrightarrow{M^{X}} / \overrightarrow{G I / 1}$ (HL or PR) priority queue, Takagi and Takahashi [18] have obtained these LSTs using the delay-cycle approach without solving such a multi-dimensional equation. However, the delay-cycle approach does not provide us the queue-length information. See also Chaudhry and Templeton [4], Meister [14], Takahashi and Takagi [20] for this situation.

Consequently, it will be useful if we get a distributional form of Little's law (abbreviated as DFLL) which gives the queue-length distribution in terms of the waiting time distribution, 
at least for the multi-class $\overrightarrow{M^{X}} / \overrightarrow{G I / 1}$ (HL) priority queue. Several previous works on DFLL are worth mentioning. Assuming Poisson arrivals, Keilson and Servi $[10,11]$ have derived a DFLL for the two-class $\overrightarrow{M / G I / 1}$ (HL) priority queue [11]. Their approach [10,11] is strongly based on the Poisson arrival assumption, and so it does not straightforwardly apply to a batch Poisson arrival queue. Assuming a renewal arrival FIFO (non-priority) queue and using the sample-path approach, Brumelle [2] has obtained the queue-length moments in terms of the waiting time and the renewal function for the arrival process, and has presented the moment-relationship between the queue-length and waiting time distributions for the Poisson arrival FIFO queue, which refines the result of Haji and Newell [6]. Note that all these previous works have treated single-arrival queue; see also Whitt [22, Section 8]. Only Brandt et al. [1] have presented a DFLL for the $M^{X} / G I / 1$ FIFO queue. However, there are few results on DFLL for batch-arrival priority queues.

This paper is organized as follows. In Section 2, we introduce notations and show a preliminary result from the stationary point process theory. Section 3 is devoted to the observation of the batch-arrival priority queue under the Palm distribution (given that a class- $p$ batch arrives at the system at time origin). In Section 4, we express the individual class queue-length distribution for the $\overrightarrow{G I^{X}} / \overrightarrow{G I / 1}$ (HL or PR) priority queue in terms of the corresponding class waiting time and completion time distributions. In Section 5, for the $\overrightarrow{M^{X}} / \overrightarrow{G I / 1}$ (HL or PR) priority queue, we obtain the z-transform for the queue-length distribution in closed form, by using the results in Sections 3 and 4 together with the LSTs for the waiting time and completion time distributions previously obtained by Takagi and Takahashi [18].

\section{The multiclass batch-arrival point processes}

Let $\mathbf{R}$ be the real space, and $\mathbf{Z}$ be the set of all integers, i.e.,

$$
\mathbf{R} \equiv(-\infty, \infty), \mathbf{Z} \equiv\{\cdots,-1,0,1,2, \cdots\}
$$

We consider a probability space $(\Omega, \mathcal{F}, P)$ with a time-shift operator group $\left\{\mathbf{T}^{s}\right\}$ on $\Omega$. Here, $\left\{\mathbf{T}^{s}\right\}$ is referred to as a time-shift operator group on $\Omega$, if for each $s \in \mathbf{R}, \mathbf{T}^{s}$ is a measurable mapping from $(\Omega, \mathcal{F})$ to $(\Omega, \mathcal{F})$, and if

$$
\mathbf{T}^{s} \circ \mathbf{T}^{t}(\omega)=\mathbf{T}^{s+t}(\omega) \quad(\omega \in \Omega ; s, t \in \mathbf{R})
$$

where o denotes the composite operator, i.e. $\mathbf{T}^{s} \circ \mathbf{T}^{t}(\omega) \equiv \mathbf{T}^{s}\left(\mathbf{T}^{t}(\omega)\right)$. Whenever no confusion occurs, we suppress argument $\omega \in \Omega$ as in the literature $[6,23]$. For example, instead of the equation above, we use

$$
\mathbf{T}^{s} \circ \mathbf{T}^{t}=\mathbf{T}^{s+t}(s, t \in \mathbf{R})
$$

Assume that $\mathrm{P}$ is stationary with respect to $\left\{\mathbf{T}^{s}\right\}$, i.e.,

$$
\left(P \circ \mathbf{T}^{s}\right)(B) \equiv P\left(\mathbf{T}^{s} B\right)=P(B) \quad(s \in \mathbf{R}, B \in \mathcal{F})
$$

where $\mathbf{T}^{s} B \equiv\left\{\mathbf{T}^{s}(\omega) \in \Omega ; \omega \in B\right\}$.

We introduce our multi-class batch-arrival process. Let $I$ be the number of classes. For each class $p(p=1,2, \cdots, I)$, let $t_{p, n}$ be the $n$-th batch epoch of class $p$, and $X_{p, n}$ be the $n$-th 
batch size (number of customers) of class $p$. We assume that for each $p(p=1,2, \cdots, I)$,

$$
\Psi_{p} \equiv\left\{\left(t_{p, n}, X_{p, n}\right)\right\}_{n=-\infty}^{+\infty}
$$

forms a stationary marked point process with respect to $P$. Here, the mark of $\Psi_{p}$ at $t_{p, n}$ is just the batch size $X_{p, n}(n \in \mathbf{Z})$. This stationarity is equivalent to assume

$$
\Psi_{p} \circ \mathbf{T}^{s}=\left\{\left(t_{p, n}-s, X_{p, n}\right)\right\}_{n=-\infty}^{+\infty} \text { for all } s \in \mathbf{R}
$$

We number those class-p customers who arrive in the $n$-th batch as $(p, n, i)(i=1,2, \cdots$, $\left.X_{p, n}\right)$. Let $t_{p, n, i}$ be the arrival epoch of a customer $(p, n, i)$. Note that $t_{p, n}=t_{p, n, i}$ for any $i\left(1 \leq i \leq X_{p, n}\right)$. We assume $\left\{t_{p, n}\right\}_{n=-\infty}^{+\infty}$ satisfies $\cdots<t_{p,-1}<t_{p, 0} \leq 0<t_{p, 1}<t_{p, 2}<\cdots$. Let $\chi_{B} \equiv \chi\{B\}$ be the indicator function of a set $B$, and let $N_{p, b}\left[\right.$ or $\left.N_{p, c}\right]$ be the point process generated by $\left\{t_{p, n}\right\}_{n=-\infty}^{+\infty}\left[\right.$ or $\left.\left\{t_{p, n, i}\right\}_{i=1}^{X_{p, n}}{ }_{n=-\infty}^{+\infty}\right]$, i.e.,

$$
\begin{aligned}
& N_{p, b}(\mathrm{~A})=\sum_{n=-\infty}^{+\infty} \chi\left\{t_{p, n} \in \mathrm{A}\right\} \quad(\mathrm{A} \in \mathcal{B}(\mathrm{R})), \\
& N_{p, c}(\mathrm{~A})=\sum_{n=-\infty}^{+\infty} \sum_{i=1}^{X_{p, n}} \chi\left\{t_{p, n, i} \in \mathrm{A}\right\}=\sum_{n=-\infty}^{+\infty} X_{p, n} \chi\left\{t_{p, n} \in \mathrm{A}\right\} \quad(\mathrm{A} \in \mathcal{B}(\mathrm{R})),
\end{aligned}
$$

where $\mathcal{B}(\mathrm{R})$ denotes a Borel field on real space $R$.

Let $E$ denote the expectation with respect to $P$. The intensities

$$
\lambda_{p, b} \equiv E N_{p, b}[0,1) \text { and } \lambda_{p, c} \equiv E N_{p, c}[0,1)
$$

are assumed to be finite and positive. Define the probability measures on $(\Omega, \mathcal{F})$ by

$$
\begin{array}{ll}
P_{p, b}(B) \equiv \frac{1}{\lambda_{p, b}} E\left[\int_{0}^{1} \chi_{B} \circ \mathbf{T}^{s} N_{p, b}(d s)\right] & (B \in \mathcal{F}), \\
P_{p, c}(B) \equiv \frac{1}{\lambda_{p, c}} E\left[\int_{0}^{1} \chi_{B} \circ \mathbf{T}^{s} N_{p, c}(d s)\right] & (B \in \mathcal{F}),
\end{array}
$$

where $\chi_{B} \circ \mathbf{T}^{s}(\omega) \equiv \chi_{B}\left(\mathbf{T}^{s} \omega\right)$. The probability measure $P_{p, b}\left[\right.$ or $\left.P_{p, c}\right]$ is called the Palm distribution with respect to $N_{p, b}$ [or $N_{p, c}$, which represents the conditional probability measure of $P$ given that a class- $P$ batch [or customer] arrives at time origin; see Franken et al. [6].

Let $E_{p, b}$ be the expectation with respect to $P_{p, b}$ and let $T_{p, n}$ the interarrival time of $(n-1)$-th and $n$-th batches $\left(T_{p, n} \equiv t_{p, n}-t_{p, n-1}\right)$. Note that in general $E\left[T_{p, 0}\right] \neq E_{p, b}\left[T_{p, 0}\right]$. Note further that $N_{p, b}$ is a simple point process, i.e., $N_{p, b}\left(\left\{t_{p, n}\right\}\right)=1(n \in \mathbf{Z})$. An inversion formula for (2.1) has been obtained as

$$
P(B)=\lambda_{p, b} E_{p, b}\left[\int_{0}^{T_{p, 1}} \chi_{B} \circ \mathrm{T}^{s} \mathrm{ds}\right] \quad(B \in \mathcal{F}),
$$

see Franken et al. [6], Miyazawa [15, 16, 17]. An inversion formula for (2.2) is similarly obtained, but we will use only (2.3) to develop our arguments.

The following lemma is a multi-class version of Lemma 3.1 of Miyazawa [16]. 
Lemma 2.1 Consider an $I$-class batch-arrival process. For a bounded random variable $u$ and any $p(p=1,2, \cdots, I)$, we have

$$
E[u]=\lambda_{p, b} E_{p, b}\left[\int_{0}^{T_{p, 1}} u \circ \mathbf{T}^{s} d s\right]
$$

Moreover, if there exists a process $\{Z(t)\}$ satisfying that $Z(s)=u \circ \mathrm{T}^{s}\left(0<s<T_{p, 1}\right)\left(a . s . P_{p, b}\right)$ and that, for each $s>0, \chi\left\{T_{p, 1}>s\right\}$ and $Z(s)$ are uncorrelated with respect to $E_{p, b}$, then

$$
E[u]=\lambda_{p, b} \int_{0}^{+\infty} P_{p, b}\left(T_{p, 1}>s\right) E_{p, b}[Z(s)] d s .
$$

Proof. Equation (2.4) is seen to be valid for any indicator function from (2.3). Any nonnegative function can be a monotone (non-decreasing) limit of these simple functions. (2.4) then follows from the monotone convergence theorem.

We then have from (2.4) and the property of $\{Z(t)\}\left[Z(s)=u \circ \mathrm{T}^{s}\left(0<s<T_{p, 1}\right)\right]$,

$$
\begin{aligned}
E[u] & =\lambda_{p, b} E_{p, b}\left[\int_{0}^{T_{p, 1}} u \circ \mathrm{T}^{s} d s\right]=\lambda_{p, b} E_{p, b}\left[\int_{0}^{\infty} \chi\left\{T_{p, 1}>s\right\} u \circ \mathrm{T}^{s} d s\right] \\
& =\lambda_{p, b} \int_{0}^{\infty} E_{p, b}\left[\chi\left\{T_{p, 1}>s\right\} Z(s)\right] d s .
\end{aligned}
$$

Since $\chi\left\{T_{p, 1}>s\right\}$ and $Z(s)$ are uncorrelated with respect to $E_{p, b}$, we have

$$
E_{p, b}\left[\chi\left\{T_{p, 1}>s\right\} Z(s)\right]=P_{p, b}\left(T_{p, 1}>s\right) E_{p, b}[Z(s)] .
$$

Substituting (2.7) into (2.6) yields (2.5).

Remark 2.2 There is a certain confusion in Lemma 3.1 of Miyazawa [16]. We here make it clear by introducing the process $\{Z(t)\}$.

\section{Key observation for the batch-arrival priority queue}

We consider a single-server priority queue with $I$-class batch arrivals. The priority is assumed to be either preemptive-resume (PR) or head-of-the-line (HL) rule. The PR priority rule permits interruptions of customers during service, and on reentering service an interrupted customer resumes service at the point of interruption; the HL rule does not permit interruptions, so that once service begins on a customer, the customer is served to completion, see e.g. $[9,23]$. We assume that a customer with a smaller index has precedence over a customer with a greater index (class 1 is the highest, and class $I$ the lowest).

For each individual class $p(p=1,2, \cdots, I)$, the service is based on the SIRO (Service-In, Random-Order) rule within a batch while it is based on the FIFO (First-In, First-Out) rule between batches. By the SIRO rule, a customer of $X_{p, n}$ is randomly selected by the server and all the selected customers of $X_{p, n}$ are numbered as $(p, n, 1),(p, n, 2), \cdots,\left(p, n, X_{p, n}\right)$; see Section 2 . The $(p, n, i)$-customer will be referred to as the $i$-th customer of batch $X_{p, n}$, and it will also be referred to as the $\left(X_{p, n}-i+1\right)$-th youngest customer of $X_{p, n}$. For example, $(p, n, 1)$-customer will be referred to as the first customer and also the $X_{p, n}$-th youngest customer, within its belonging batch $X_{p, n}$. 
For any $p(1 \leq p \leq I), n \in \mathrm{Z}$ and $i\left(1 \leq i \leq X_{p, n}\right)$, let $S_{p, n, i}$ be the service time of a $(p, n, i)$-customer. We assume that

$$
\Phi_{p} \equiv\left\{\left(t_{p, n}, X_{p, n}, S_{p, n, 1}, S_{p, n, 2}, \cdots, S_{p, n, X_{p, n}}\right)\right\}_{n=-\infty}^{+\infty}
$$

forms a stationary marked point process with respect to $P$. Here, the mark of $\Phi_{p}$ at $t_{p, n}$ is the $\left(X_{p, n}+1\right)$-dimensional vector $\left(X_{p, n}, S_{p, n, 1}, S_{p, n, 2}, \cdots, S_{p, n, X_{p, n}}\right)$. This assumption is equivalent to

$$
\Phi_{p} \circ \mathrm{T}^{s}=\left\{\left(t_{p, n}-s, X_{p, n}, S_{p, n, 1}, S_{p, n, 2}, \cdots, S_{p, n, X_{p, n}}\right)\right\}_{n=-\infty}^{+\infty} \text { for all } s \in \mathbf{R} .
$$

We further assume that the batch interarrival sequence $\left\{T_{p, n}\right\}_{n=-\infty}^{+\infty}$, the batch size sequence $\left\{X_{p, n}\right\}_{n=-\infty}^{+\infty}$, and the service time sequence $\left\{S_{p, n, i}\right\}_{i=1}^{X_{p, n}+\infty}+{ }_{n=-\infty}^{+\infty}$ are respectively i.i.d. and mutually independent with respect to $P_{p, b}$. Since $S_{p, 0, i} \circ \mathrm{T}^{s}=S_{p, 0, i}, X_{p, 0} \circ \mathrm{T}^{s}=X_{p, 0}$ for $0<s<T_{p, 1}\left(\right.$ a.s. $\left.P_{p, b}\right)$, note that the sequences $\left\{X_{p, n}\right\}_{n=-\infty}^{+\infty}$, and $\left\{S_{p, n, i}\right\}_{i=1}^{X_{p, n}}{ }_{n=-\infty}^{+\infty}$ are respectively i.i.d. and independent of $\left\{T_{p, n}\right\}_{n=-\infty}^{+\infty}$ with respect to $P$. Note also that $E_{p, b}\left(X_{p, 0}\right)=E\left(X_{p, 0}\right)$, and $E_{p, b}\left(S_{p, 0, i}\right)=E\left(S_{p, 0, i}\right) ;$ see Appendix.

Moreover, we assume that $\Phi_{p}$ and $\Phi_{q}$ are independent with respect to $P$ for $p \neq q(1 \leq$ $p, q \leq I)$, as usual in the literature of priority queues [9, 23]. This assumption excludes the structured input priority queues treated by Takahashi et al. [20,21]. From now on, our input will be denoted by $\sum_{p=1}^{I} \Phi_{p}$ or $\overrightarrow{G I^{X}} / \overrightarrow{G I}$ as in König et al. [13] and our single-server system by the $\overrightarrow{G I^{X}} / \overrightarrow{G I / 1}$ (HL or PR) priority queue.

Here are basic notations for a class $p(p=1,2, \cdots, I)$ in the $\overrightarrow{G I^{X}} / \overrightarrow{G I} / 1$ (HL or PR) priority queue.

$l_{p}(\mathrm{t}):$ the number of customers in the system (queue-length) at time $t$

$q_{p}(\mathrm{t})$ : the number of customers in the waiting room (wait-length) at time $t$

and

$W_{p, b, n}$ : the waiting time of the first customer in the $n$-th arriving batch.

We use the completion time to study the priority queue as in Jaiswal [9]. Consider a $(p, n, i)$-customer and the next class- $p$ customer. Here, the next class- $p$ customer is the one with index $(p, n, i+1)$ if $i<X_{p, n}$, and $(p, n+1,1)$ if $i=X_{p, n}$. The completion time $C_{p, n, i}$ of a $(p, n, i)$-customer is then defined as the time duration of a period that begins at the instant when the service of a $(p, n, i)$-customer starts and ends at the instant when the server becomes free to take the next class- $p$ customer. In other words, the completion time $C_{p, n, i}$ is the interval between the moment at which a $(p, n, i)$-customer enters service and the first moment at which there are no higher class $\{1,2, \cdots, p-1\}$ customers in the system after the service of this $(p, n, i)$-customer. Note that the completion time $C_{1, n, i}$ for the highest priority class $(p=1)$ equals the service time $S_{1, n, i}$ under both PR and HL priority rules. Note also that the completion time $C_{p, n, i}$ under the PR rule is identical to that under the HL rule; see e.g. [21, Theorem 3.4].

The total traffic intensity given by

$$
\rho \equiv \sum_{p=1}^{I} \lambda_{p, c} E\left[S_{p, n, i}\right]=\sum_{p=1}^{I} \lambda_{p, b} E\left[X_{p, n}\right] E\left[S_{p, n, i}\right]
$$


is assumed to be less than unity. Then, from the stationarity assumption on $\Phi_{p}$, we can construct for each class $p$, the queue-length process $l_{p}(t)$ and the wait-length process $q_{p}(t)$ which are stationary with respect to $P$, i.e., the processes $\left\{l_{p}(t)\right\}$ and $\left\{q_{p}(t)\right\}$ respectively satisfy

$$
l_{p}(s)=l_{p}(0) \circ \mathbf{T}^{s}, \quad q_{p}(s)=q_{p}(0) \circ \mathbf{T}^{s} \quad(p=1,2, \cdots, I) .
$$

Moreover, the waiting time sequence of the first customer in a batch $\left\{W_{p, b, n}\right\}$ and the completion time sequence $\left\{C_{p, n, i}\right\}$ are stationary with respect to $P_{p, b}$. See Franken et al [6] and Miyazawa [15] for the construction of these stationary processes $\left\{l_{p}(t)\right\},\left\{q_{p}(t)\right\},\left\{W_{p, b, n}\right\}$, and $\left\{C_{p, n, i}\right\}$.

Consider an event $\left\{l_{p}(s) \geq j\right\}$ for $j \geq 0$ and $s\left(0<s<t_{p, 1}\right)$ in the queue under the measure $P_{p, b}(p=1,2, \cdots, I)$. Namely, we consider the conditional event $\left\{l_{p}(s) \geq j\right\}$, given that a class- $p$ batch arrived at the queue at the the origin of time axis. Recalling the definition of $\left\{t_{p, n}\right\}_{n=-\infty}^{+\infty}$ in Section 2, we note that $t_{p, 0}=0$, and $t_{p, 1}=T_{p, 1}\left(\right.$ a.s. $\left.P_{p, b}\right)$. For $s<t_{p, 1}$, there are no arrivals of batches during $(0, s)$, so that all the customers in the system at time $s$ arrived at or before time origin. Hence, if we introduce:

$$
\begin{aligned}
Z S_{p}(j, s) \equiv & \{\text { the } j \text {-th youngest class-p customer who arrived during }(-\infty, 0] \\
& \text { still remains in the system at time } \mathrm{s}\}
\end{aligned}
$$

we have

$$
\left\{l_{p}(s) \geq j\right\}=Z S_{p}(j, s) \quad\left(0<s<T_{p, 1}\right) \quad\left(\text { a.s. } P_{p, b}\right) .
$$

Let $-i(i \geq 0)$ denote the batch index of the $j$-th youngest class- $p$ customer among the customers who are in the system at time $s$, i.e.,

$$
X_{p,-i}+X_{p,-i+1}+\cdots+X_{p, 0} \geq j>X_{p,-i+1}+\cdots+X_{p, 0}
$$

If we set $X_{p,-i+1}+\cdots+X_{p, 0}=k$ and $X_{p,-i}=m$, it is easily verified from these definitions of $i, k$, and $m$, that the $j$-th youngest customer who arrived during $(-\infty, 0]$ is just the $(j-k)$-th youngest customer of its belonging batch $X_{p,-i}$. By (3.3) we introduce:

$$
B_{p}(j, i, k, m) \equiv\left\{k+m \geq j>k, X_{p,-i+1}+\cdots+X_{p, 0}=k \text { and } X_{p,-i}=m\right\} .
$$

For convenience' sake we further introduce:

$$
\begin{gathered}
R S_{p}(j, i, k, m, s) \equiv\left\{\text { the }(j-k) \text {-th youngest customer of } X_{p,-i}\right. \text { still remains } \\
\text { in the system at time } s\} .
\end{gathered}
$$

It then follows that

$$
P_{p, b}\left\{Z S_{p}(j, s) \mid B_{p}(i, k, m)\right\}=P_{p, b}\left\{R S_{p}(j, i, k, m, s) \mid B_{p}(j, i, k, m)\right\}
$$

and

$$
P_{p, b}\left\{Z S_{p}(j, s)\right\}=\sum_{i=0}^{\infty} \sum_{k=0}^{j-1} \sum_{m=j-k}^{\infty} P_{p, b}\left\{R S_{p}(j, i, k, m, s) \mid B_{p}(j, i, k, m)\right\} P_{p, b}\left\{B_{p}(j, i, k, m)\right\} .
$$

We next consider each conditional probability $P_{p, b}\left\{R S_{p}(j, i, k, m, s) \mid B_{p}(j, i, k, m)\right\}$ appearing in (3.7) under the HL or PR priority rule, respectively. 
Under the HL rule, a customer in service is not interrupted and the customer departure epoch corresponds to the end of its service time. It then follows that the first [ $m$-th youngest] customer of $X_{p,-i}$ still remains in the system at time $s$, if and only if the sum $W_{p,-i}+S_{p,-i, 1}$ exceeds $T_{p,-i+1}+\cdots+T_{p, 0}+s$; see Figure 1 . This follows from the facts that the system sojourn time for the first customer equals $W_{p,-i}+S_{p,-i, 1}$ and that its sojourn time exceeds $T_{p,-i+1}+\cdots+T_{p, 0}+s$ iff the first customer remains in the system at time s. Here, iff denotes if and only if.

In the same way it follows that the second [( $m-1)$-th youngest] customer of $X_{p,-i}$ still remains in the system, iff the sum $W_{p,-i}+C_{p,-i, 1}+S_{p,-i, 2}$ exceeds $T_{p,-i+1}+\cdots+T_{p, 0}+s$. The third customer $[(m-2)$-th youngest $]$ of $X_{p,-i}$ still remains in the system, iff the sum $W_{p,-i}+C_{p,-i, 1}+C_{p,-i, 2}+S_{p,-i, 3}$ exceeds $T_{p,-i+1}+\cdots+T_{p, 0}+s$. Therefore, it follows that the $(m-j+k+1)$-th $\left[(j-k)\right.$-th youngest] customer of $X_{p,-i}$ still remains in the system, iff the sum $W_{p,-i}+C_{p,-i, 1}+\cdots+C_{p,-i, m-j+k}+S_{p,-i, m-j+k+1}$ exceeds $T_{p,-i+1}+\cdots+T_{p, 0}+s$. This observation leads to the following equality.

$$
\begin{aligned}
P_{p, b}\left\{R S_{p}(j, i, k, m, s) \mid\right. & \left.B_{p}(j, i, k, m)\right\} \\
& =P_{p, b}\left\{W_{p,-i}+C_{p,-i, 1}+\cdots+C_{p,-i, m-j+k}+S_{p,-i, m-j+k+1}\right. \\
& \left.>T_{p,-i+1}+\cdots+T_{p, 0}+s\right\} \quad \text { under HL. }
\end{aligned}
$$

Here, we use the convention for empty sum, e.g. $C_{p,-i, 1}+\cdots+C_{p,-i, 0}=0$.

Under the PR priority rule, a customer in service can be interrupted and the customer departure epoch corresponds to the end of its completion time. A similar argument to deriving (3.8) yields that the $(j-k)$-th youngest customer of $X_{p,-i}$ still remains in the system, iff the sum of the waiting time of the first customer of the $i$-th batch $\left(W_{p,-i}\right)$ and $m-(j-k)+1$ completion times $\left(C_{p,-i, 1}+\cdots+C_{p,-i, m-j+k}+C_{p,-i, m-j+k+1}\right)$ exceeds the time interval $T_{p,-i+1}+\cdots+T_{p, 0}+s$, i.e.,

$$
\begin{aligned}
P_{p, b}\left\{R S_{p}(j, i, k, m, s) \mid\right. & \left.B_{p}(j, i, k, m)\right\} \\
& =P_{p, b}\left\{W_{p,-i}+C_{p,-i, 1}+\cdots+C_{p,-i, m-j+k}+C_{p,-i, m-j+k+1}\right. \\
& \left.>T_{p,-i+1}+\cdots+T_{p, 0}+s\right\} \quad \text { under PR. }
\end{aligned}
$$

We are in a position to consider an event $\left\{q_{p}(s) \geq j\right\}$ for $j \geq 0$ and $s\left(0<s<t_{p, 1}\right)$. We also introduce:

$$
\begin{aligned}
& Z Q_{p}(j, s) \equiv\{\text { the } j \text {-th youngest class-p customer who arrived during }(-\infty, 0] \\
& \text { still remains in the waiting room at time } s\} ;
\end{aligned}
$$$$
R Q_{p}(j, i, k, m, s) \equiv\left\{\text { the }(j-k) \text {-th youngest customer of } X_{p,-i}\right. \text { still remains }
$$$$
\text { in the waiting room at time } s\} \text {. }
$$

Regarding the waiting room as the whole system, we have

$$
\begin{gathered}
\left\{q_{p}(s) \geq j\right\}=Z Q_{p}(j, s) \quad\left(0<s<T_{p, 1}\right) \\
P_{p, b}\left\{Z Q_{p}(j, s) \mid B_{p}(j, i, k, m)\right\}=P_{p, b}\left\{R Q_{p}(j, i, k, m, s) \mid B_{p}(j, i, k, m)\right\} \\
P_{p, b}\left\{Z Q_{p}(j, s)\right\}=\sum_{i=0}^{\infty} \sum_{k=0}^{j-1} \sum_{m=j-k}^{\infty} P_{p, b}\left\{R Q_{p}(j, i, k, m, s) \mid B_{p}(j, i, k, m)\right\} P_{p, b}\left\{B_{p}(j, i, k, m)\right\} .
\end{gathered}
$$


Under the HL priority rule, the discussions for $P_{p, b}\left\{R S_{p}(j, i, k, m, s) \mid B_{p}(i, k, m)\right\}$ can be applied for $P_{p, b}\left\{R Q_{p}(j, i, k, m, s) \mid B_{p}(j, i, k, m)\right\}$ to obtain the following equality.

$$
\begin{aligned}
P_{p, b} & \left\{R Q_{p}(j, i, k, m, s) \mid B_{p}(j, i, k, m)\right\}=P_{p, b}\left\{W_{p,-i}+C_{p,-i, 1}+\cdots+C_{p,-i, m-(j-k)}\right. \\
> & \left.T_{p,-i+1}+\cdots+T_{p, 0}+s\right\} \quad \text { under HL. }
\end{aligned}
$$

Under the PR rule it is difficult to obtain a similar equation to (3.13) because of the preemptions. However, if we distinguish between the number of customers in the waiting room and that in limbo [21] (the waiting room is used for unserved customers while limbo is used for interrupted customers), (3.13) is seen to also be valid under PR by the same observation. Henceforth, when considering the PR rule, we will draw this distinction and define the queue-length as the number of customers in the waiting room, so that

$$
\begin{gathered}
P_{p, b}\left\{R Q_{p}(j, i, k, m, s) \mid B_{p}(j, i, k, m)\right\}=P_{p, b}\left\{W_{p,-i}+C_{p,-i, 1}+\cdots+C_{p,-i, m-j+k}\right. \\
\left.>T_{p,-i+1}+\cdots+T_{p, 0}+s\right\} \quad \text { under PR. }
\end{gathered}
$$

We summarize the results obtained above [(3.2) through (3.14)] in the following lemma.

Lemma 3.1 In a $\overrightarrow{G I^{X}} / \overrightarrow{G I / 1}$ (HL or PR) priority queue, we have for an individual class $p$ (i) $P_{p, b}\left\{Z S_{p}(j, s)\right\}$ is given by

$$
\begin{aligned}
\sum_{i=0}^{\infty} \sum_{k=0}^{j-1} \sum_{m=j-k}^{\infty} & P_{p, b}\left\{W_{p,-i}+C_{p,-i, 1}+\cdots+C_{p,-i, m-j+k}+S_{p,-i, m-j+k+1}\right. \\
& \left.>T_{p,-i+1}+\cdots+T_{p, 0}+s\right\} \\
& \times P_{p, b}\left\{X_{p,-i+1}+\cdots+X_{p, 0}=k, \text { and } X_{p,-i}=m\right\} \quad \text { under HL }
\end{aligned}
$$

and

$$
\begin{aligned}
\sum_{i=0}^{\infty} \sum_{k=0}^{j-1} \sum_{m=j-k}^{\infty} P_{p, b} & \left\{W_{p,-i}+C_{p,-i, 1}+\cdots+C_{p,-i, m-j+k}+C_{p,-i, m-j+k+1}\right. \\
& \left.>T_{p,-i+1}+\cdots+T_{p, 0}+s\right\} \\
& \times P_{p, b}\left\{X_{p,-i+1}+\cdots+X_{p, 0}=k, \text { and } X_{p,-i}=m\right\} \quad \text { under PR. }
\end{aligned}
$$

(ii) $P_{p, b}\left\{Z Q_{p}(j, s)\right\}$ is given by

$$
\begin{aligned}
\sum_{i=0}^{\infty} \sum_{k=0}^{j-1} \sum_{m=j-k}^{\infty} P_{p, b}\left\{W_{p,-i}+C_{p,-i, 1}+\cdots+C_{p,-i, m-j+k}>T_{p,-i+1}+\cdots+T_{p, 0}+s\right\} \\
\times P_{p, b}\left\{X_{p,-i+1}+\cdots+X_{p, 0}=k \text {, and } X_{p,-i}=m\right\} \quad \text { under HL or PR. }
\end{aligned}
$$

4. The queue-length distribution for the $\overrightarrow{G I^{X}} / \overrightarrow{G I / 1}$ (HL or PR) priority queue From the results in Section 3 it follows that $T_{p, 1}$ is independent of the event $Z S_{p}(j, s)$ [or $\left.Z Q_{p}(j, s)\right]$ with respect to $P_{p, b}$. Indeed, for our $\overrightarrow{G I^{X}} / \overrightarrow{G I / 1}$ (HL or PR) priority queue, recall that $T_{p, 1}$ is independent of $\left\{T_{p, n}\right\}_{n=-i}^{0},\left\{X_{p, n}\right\}_{n=-i}^{0}, W_{p,-i},\left\{S_{p,-i, n}\right\}_{n=1}^{X_{p,-i}}$, and $\left\{C_{p,-i, n}\right\}_{n=1}^{X_{p,-i}}$ for all $i \geq 0$ with respect to $P_{p, b}$. From (3.8) and (3.9) [or (3.13) and (3.14)], it is independent 
of the events $B_{p}(j, i, k, m)$ and $R S_{p}(j, i, k, m, s)$ [or $\left.R Q_{p}(j, i, k, m, s)\right]$. Thus, from (3.7) [or (3.12)], we see that $T_{p, 1}$ is independent of $Z S_{p}(j, s)$ [or $\left.Z Q_{p}(j, s)\right]$ with respect to $P_{p, b}$.

For an indicator function $u=\chi\left\{l_{p}(0) \geq j\right\}$ or $\chi\left\{q_{p}(0) \geq j\right\}$, it follows from (3.1) that $u \circ \mathbf{T}^{s}=\chi\left\{l_{p}(s) \geq j\right\}$ or $\chi\left\{q_{p}(s) \geq j\right\}$. It then follows from (3.2) and (3.10)

$$
u \circ \mathbf{T}^{s}=\chi\left\{Z S_{p}(j, s)\right\} \text { or } u \circ \mathbf{T}^{s}=\chi\left\{Z Q_{p}(j, s)\right\}\left(0<s<T_{p, 1}\right) \quad\left(\text { a.s. } P_{p, b}\right) .
$$

Since $T_{p, 1}$ is independent of the events $Z S_{p}(j, s)$ and $Z Q_{p}(j, s)$ as seen above, for each $s>0 \chi\left\{T_{p, 1}>s\right\}$ and $\chi\left\{Z S_{p}(j, s)\right\}\left[\right.$ or $\left.\chi\left\{Z Q_{p}(j, s)\right\}\right]$ are uncorrelated with respect to $E_{p, b}$. Namely, both the process $\left\{Z S_{p}(j, t)\right\}$ and $\left\{Z Q_{p}(j, t)\right\}$ satisfy the condition for $\{Z(t)\}$ in Lemma 2.1. Applying Lemma 2.1 with indicator function $u=\chi\left\{l_{p}(0) \geq j\right\}$ or $\chi\left\{q_{p}(0) \geq j\right\}$, we obtain the following lemma.

Lemma 4.1 In a $\overrightarrow{G I^{X}} / \overrightarrow{G I / 1}$ (HL or PR) priority queue, we have for an individual class $p$

$$
P\left(l_{p} \geq j\right) \equiv P\left(l_{p}(0) \geq j\right)=\lambda_{p, b} \int_{0}^{+\infty} P_{p, b}\left(T_{p, 1}>s\right) P_{p, b}\left(Z S_{p}(j, s)\right) d s(j \geq 0),
$$

and

$$
P\left(q_{p} \geq j\right) \equiv P\left(q_{p}(0) \geq j\right)=\lambda_{p, b} \int_{0}^{+\infty} P_{p, b}\left(T_{p, 1}>s\right) P_{p, b}\left(Z Q_{p}(j, s)\right) d s(j \geq 0),
$$

where $l_{p}$ and $q_{p}$ denote the stationary queue-length and the wait-length, respectively.

To obtain the queue-length and wait-length distributions in terms of the waiting time and completion times, we also need the following lemma.

Lemma 4.2 For a non-negative real-valued random variable $Y$ (with respect to $P_{p, b}$ ), if $Y$ is independent of $\left\{T_{p,-i+1}, \cdots, T_{p, 0}\right\}$, we have

$$
\begin{aligned}
\int_{0}^{+\infty} P_{p, b}\left(T_{p, 1}>s\right) & P_{p, b}\left(Y>T_{p,-i+1}+\cdots+T_{p, 0}+s\right) d s \\
& =\int_{0}^{+\infty} P_{p, b}(Y>s) P_{p, b}\left(N_{p, b}(0, s)=i\right) d s \quad(p=1, \cdots, I) .
\end{aligned}
$$

Proof. From the definitions of $\left\{T_{p, i}\right\}$ and $N_{p, b}(0, t)$, we have

$$
\begin{aligned}
\int_{s=0}^{+\infty} P_{p, b} & \left(T_{p, 1}>s\right) P_{p, b}\left(Y>T_{p,-i+1}+\cdots+T_{p, 0}+s\right) d s \\
& =\int_{x=0}^{+\infty} \int_{s=0}^{+\infty} P_{p, b}\left(T_{p, 1}>s\right) P_{p, b}(Y>x+s) d_{x} P_{p, b}\left(T_{p,-i+1}+\cdots+T_{p, 0} \leq x\right) d s
\end{aligned}
$$

[by setting $u=x+s$ ]

$$
\begin{aligned}
& =\int_{x=0}^{+\infty} \int_{u=x}^{+\infty} P_{p, b}\left(T_{p, 1}>u-x\right) P_{p, b}(Y>u) d_{x} P_{p, b}\left(T_{p,-i+1}+\cdots+T_{p, 0} \leq x\right) d u \\
& =\int_{u=0}^{+\infty} P_{p, b}(Y>u) \int_{x=0}^{u} P_{p, b}\left(T_{p, 1}>u-x\right) d_{x} P_{p, b}\left(T_{p,-i+1}+\cdots+T_{p, 0} \leq x\right) d u \\
& =\int_{u=0}^{+\infty} P_{p, b}(Y>u) P_{p, b}\left(T_{p,-i+1}+\cdots+T_{p, 0} \leq u<T_{p,-i+1}+\cdots+T_{p, 0}+T_{p, 1}\right) d u
\end{aligned}
$$


[by the stationarity of $\left\{T_{p, n}\right\}$ with respect to $P_{p, b}$ ]

$$
\begin{aligned}
& =\int_{u=0}^{+\infty} P_{p, b}(Y>u) P_{p, b}\left(T_{p, 1}+\cdots+T_{p, i} \leq u<T_{p, 1}+\cdots+T_{p, i}+T_{p, i+1}\right) d u \\
& =\int_{u=0}^{+\infty} P_{p, b}(Y>u) P_{p, b}\left(N_{p, b}(0, u)=i\right) d u,
\end{aligned}
$$

which completes the proof.

We use the following probability generating functions $(p g f s)$ and Laplace-Stieltjes transforms (LSTs) defined on complex number $z$ or $\theta[|z| \leq 1, \operatorname{Re}(\theta)>0]$ for class $p(p=$ $1,2, \cdots, I)$.

$\tilde{X}_{p}(z) \equiv E_{p, b}\left(z^{X_{p, n}}\right)$ (pgf for the batch size distribution)

$S_{p}^{*}(\theta) \equiv E_{p, c}\left(e^{-\theta S_{p, n, i}}\right)$ (LST for the service-time distribution)

$\tilde{l}_{p}(z) \equiv E\left(z^{l_{p}(t)}\right)$ (pgf for the stationary queue-length distribution)

$\tilde{q}_{p}(z) \equiv E\left(z^{q_{p}(t)}\right)$ (pgf for the stationary wait-length distribution)

$W_{p, b}^{*}(\theta) \equiv E_{p, b}\left(e^{-\theta W_{p, b, n}}\right)$ (LST for the stationary waiting-time distribution of a $(p, n, 1)$ customer)

and

$C_{p}^{*}(\theta) \equiv E_{p, b}\left(e^{-\theta C_{p, n, i}}\right)$ (LST for the completion time distribution of a $(p, n, i)$-customer).

We are now in a position to derive the $z$-transforms of the queue-length and the waitlength distributions in terms of the completion times as well as the waiting time of the first customer in a batch.

Theorem 4.3 Consider a batch-arrival single-server $\left(\overrightarrow{G I^{X}} / \overrightarrow{G I / 1}\right)$ priority queue with $I$ classes. For an individual class $p(1 \leq p \leq I)$, we have

$$
\begin{aligned}
\tilde{l}_{p}(z) & =1-\frac{1-z}{z} \lambda_{p, b} \sum_{m=1}^{\infty} \sum_{j=1}^{m} \int_{0}^{+\infty} P_{p, b}\left(W_{p, b, 0}+C_{p, 0,1}+\cdots+C_{p, 0, m-j}+S_{p, 0, m-j+1}>s\right) z^{j} \\
& \times P_{p, b}\left(X_{p, 0}=m\right) E_{p, b}\left(\tilde{X}_{p}(z)^{N_{p, b}(0, s)}\right) d s \quad \text { under HL, } \\
\tilde{l}_{p}(z) & =1-\frac{1-z}{z} \lambda_{p, b} \sum_{m=1}^{\infty} \sum_{j=1}^{m} \int_{0}^{+\infty} P_{p, b}\left(W_{p, b, 0}+C_{p, 0,1}+\cdots+C_{p, 0, m-j}+C_{p, 0, m-j+1}>s\right) z^{j} \\
& \times P_{p, b}\left(X_{p, 0}=m\right) E_{p, b}\left(\tilde{X}_{p}(z)^{N_{p, b}(0, s)}\right) d s \quad \text { under PR, }
\end{aligned}
$$

and

$$
\begin{aligned}
\tilde{q}_{p}(z) & =1-\frac{1-z}{z} \lambda_{p, b} \sum_{m=1}^{\infty} \sum_{j=1}^{m} \int_{0}^{+\infty} P_{p, b}\left(W_{p, b, 0}+C_{p, 0,1}+\cdots+C_{p, 0, m-j}>s\right) z^{j} \\
& \times P_{p, b}\left(X_{p, 0}=m\right) E_{p, b}\left(\tilde{X}_{p}(z)^{N_{p, b}(0, s)}\right) d s \quad \text { under HL or PR. }
\end{aligned}
$$

Proof. We first prove (4.5) assuming the PR priority rule. It follows from (i) of Lemma 3.1 and (4.1) that

$$
\begin{aligned}
P\left(l_{p} \geq j\right)=\lambda_{p, b} & \sum_{i=0}^{+\infty} \sum_{k=0}^{j-1} \sum_{m=j-k}^{+\infty} \int_{0}^{+\infty} P_{p, b}\left(T_{p, 1}>s\right) \\
& \times P_{p, b}\left(W_{p,-i}+C_{p,-i, 1}+\cdots+C_{p,-i, m-j+k+1}>T_{p,-i+1}+\cdots+T_{p, 0}+s\right) d s \\
& \times P_{p, b}\left(X_{p,-i}=m\right) P_{p, b}\left(X_{p,-i+1}+\cdots+X_{p, 0}=k\right)
\end{aligned}
$$


[by Lemma 4.2]

$$
\begin{aligned}
& =\lambda_{p, b} \sum_{i=0}^{+\infty} \sum_{k=0}^{j-1} \sum_{m=j-k}^{+\infty} \int_{0}^{+\infty} P_{p, b}\left(W_{p,-i}+C_{p,-i, 1}+\cdots+C_{p,-i, m-j+k+1}>s\right) P_{p, b}\left(N_{p, b}(0, s)=i\right) d s \\
& \times P_{p, b}\left(X_{p,-i}=m\right) P_{p, b}\left(X_{p,-i+1}+\cdots+X_{p, 0}=k\right) \\
& =\lambda_{p, b} \sum_{i=0}^{+\infty} \sum_{k=0}^{j-1} \sum_{m=j-k}^{+\infty} \int_{0}^{+\infty} P_{p, b}\left(W_{p, 0}+C_{p, 0,1}+\cdots+C_{p, 0, m-j+k+1}>s\right) P_{p, b}\left(N_{p, b}(0, s)=i\right) d s \\
& \times P_{p, b}\left(X_{p, 0}=m\right) P_{p, b}\left(X_{p, 1}+\cdots+X_{p, i}=k\right) .
\end{aligned}
$$

Here, we used the stationarity of $\left\{W_{p, n}\right\},\left\{C_{p, n, i}\right\}$, and $\left\{X_{p, n}\right\}$ with respect to $P_{p, b}$. Multiplying both sides of $(4.7)$ by $z^{j}$, summing up with respect to $j(j \geq 1)$, and changing the orders of the summation as

$$
\sum_{j=1}^{\infty} \sum_{i=0}^{\infty} \sum_{k=0}^{j-1} \sum_{m=j-k}^{\infty}=\sum_{i=0}^{\infty} \sum_{k=0}^{\infty} \sum_{j=k+1}^{\infty} \sum_{m=j-k}^{\infty}=\sum_{i=0}^{\infty} \sum_{k=0}^{\infty} \sum_{m=1}^{\infty} \sum_{u=1}^{m}(\text { where } u \equiv j-k),
$$

we obtain

$$
\begin{aligned}
\sum_{j=1}^{\infty} P\left(l_{p} \geq j\right) z^{j} & =\frac{z\left[1-\tilde{l}_{p}(z)\right]}{1-z} \\
& =\lambda_{p, b} \sum_{m=1}^{\infty} \sum_{u=1}^{m} \int_{0}^{+\infty} P_{p, b}\left(W_{p, 0}+C_{p, 0,1}+\cdots+C_{p, 0, m-u+1}>s\right) z^{u} \\
& \times P_{p, b}\left(X_{p, 0}=m\right) E_{p, b}\left(\tilde{X}_{p}(z)^{N_{p, b}(0, s)}\right) d s
\end{aligned}
$$

which yields (4.5).

Under the HL priority rule, (4.4) follows if we replace $C_{p,-i, m-j+k+1}$ by $S_{p,-i, m-j-k+1}$ in the discussion above.

Similarly, (4.6) follows from (ii) of Lemma 3.1 and (4.2). This completes the proof.

\section{DFLL for the batch Poisson arrival $\overrightarrow{M^{X}} / \overrightarrow{G I / 1}$ (HL or PR) priority queue}

Let us consider a batch Poisson arrival $\overrightarrow{M^{X}} / \overrightarrow{G I / 1}$ (HL or PR) priority queue with $I$ classes, where the interarrival times of individual class batches are independent, and exponentially distributed. For convenience, the set of all customers included a batch is called a supercustomer. The LST for the service time distribution of a class- $p$ supercustomer is given by $\tilde{X}_{p}\left(S_{p}^{*}(\theta)\right)$ with mean $E\left[X_{p, n}\right] E\left[S_{p, n, i}\right]$. Let $\lambda_{p, b}^{+}$be the arrival rate of supercustomers of class $\{1,2, \cdots, p\}$, i.e.,

$$
\lambda_{p, b}^{+}=\sum_{k=1}^{p} \lambda_{k, b}=\sum_{k=1}^{p} E N_{k, b}[0,1) .
$$

The $\operatorname{LST} B_{p, b}^{+}(\theta)$ for the service time distribution of a supercustomer of class $\{1,2, \cdots, p\}$ is then given by

$$
B_{p, b}^{+}(\theta)=\frac{1}{\lambda_{p, b}^{+}} \sum_{k=1}^{p} \lambda_{k, b} \tilde{X}_{k}\left(S_{k}^{*}(\theta)\right)
$$

Let $\Theta_{p, b}^{+}(\theta)$ be the LST of the busy period length distribution for supercustomers of class $\{1,2, \cdots, p\}$. It is well known that the $\operatorname{LST} \Theta_{p, b}^{+}(\theta)$ satisfies the equation:

$$
\Theta_{p, b}^{+}(\theta)=B_{p, b}^{+}\left(\theta+\lambda_{p, b}^{+}-\lambda_{p, b}^{+} \Theta_{p, b}^{+}(\theta)\right)
$$


If we introduce:

$$
\sigma_{p} \equiv \sigma_{p}(\theta) \equiv \theta+\lambda_{p, b}^{+}-\lambda_{p, b}^{+} \Theta_{p, b}^{+}(\theta)
$$

the $\operatorname{LST} C_{p}^{*}(\theta)$ for the completion time distribution of a $(p, n . i)$-customer is obtained as

$$
C_{p}^{*}(\theta)=S_{p}^{*}\left(\sigma_{p-1}\right)
$$

Similarly, the $\operatorname{LST} C_{p, b}^{*}(\theta)$ for the completion time distribution of a class- $p$ supercustomer is obtained as

$$
C_{p, b}^{*}(\theta)=\tilde{X}_{p}\left(S_{p}^{*}\left(\sigma_{p-1}\right)\right)
$$

Using the delay-cycle approach as in Kella and Yechiali [12], Takagi et al. [18] and Takahashi et al. $[20,21]$ have obtained the $\operatorname{LST} W_{p, b}^{*}(\theta)$ for the waiting-time distribution of the first customer in a class- $p$ batch as follows:

$$
W_{p, b}^{*}(\theta)=\frac{(1-\rho) \sigma_{p-1}+\sum_{k=p+1}^{I} \lambda_{k, b} E\left[X_{k}\right]\left(1-S_{p}^{*}\left(\sigma_{p-1}\right)\right)}{\theta-\lambda_{p, b}+\lambda_{p, b} \tilde{X}_{p}\left(S_{p}^{*}\left(\sigma_{p-1}\right)\right)}
$$

or

$$
W_{p, b}^{*}(\theta)=\frac{(1-\rho) \sigma_{p-1}+\sum_{k=p+1}^{I} \lambda_{k, c}\left(1-C_{p}^{*}(\theta)\right)}{\theta-\lambda_{p, b}+\lambda_{p, b} C_{p, b}^{*}(\theta)},
$$

in terms of the LSTs for the completion times $C_{p}^{*}(\theta)$ and $C_{p, b}^{*}(\theta)$.

We are finally in a position to derive our DFLL (distributional form of Little's law). Note that

$$
E_{p, b}\left(\tilde{X}_{p}(z)^{N_{p, b}(0, s)}\right)=e^{-s \lambda_{p, b}\left(1-\tilde{X}_{p}(z)\right)} \quad(p=1,2, \cdots, I) .
$$

Note also that for a non-negative real-valued random variable $R$ and a complex number $\gamma$ with positive real part

$$
\int_{0}^{+\infty} P_{p, b}(R>s) e^{-s \gamma} \mathrm{ds}=\frac{1-R^{*}(\gamma)}{\gamma}
$$

where $R^{*}(\theta) \equiv \int_{0}^{+\infty} e^{-\theta t} d_{t} P_{p, b}(R \leq t)$.

Let us introduce the following notation for simplicity.

$$
\eta_{p} \equiv \eta_{p}(z) \equiv \lambda_{p, b}\left(1-\tilde{X}_{p}(z)\right)
$$

Applying Theorem 4.3 leads to the following relationship between the queue-length and the waiting time distributions, which can be regarded as a DFLL.

Theorem 5.1 Consider a batch Poisson arrival $\left(\overrightarrow{M^{X}} / \overrightarrow{G I / 1}\right)$ priority queue with $I$ classes. For an individual class $p(1 \leq p \leq I)$, we have

$$
\begin{aligned}
& \tilde{l}_{p}(z)=\tilde{q}_{p}(z) S_{p}^{*}\left(\eta_{p}\right) \quad \text { under HL, } \\
& \tilde{l}_{p}(z)=\tilde{q}_{p}(z) C_{p}^{*}\left(\eta_{p}\right) \quad \text { under PR, }
\end{aligned}
$$

and

$$
\tilde{q}_{p}(z)=\frac{1-z}{1-\tilde{X}_{p}(z)} \cdot \frac{\tilde{X}_{p}\left(C_{p}^{*}\left(\eta_{p}\right)\right)-\tilde{X}_{p}(z)}{C_{p}^{*}\left(\eta_{p}\right)-z} \cdot W_{p, b}^{*}\left(\eta_{p}\right) \quad \text { under HL or PR. }
$$


Here, $\eta_{p}$ is defined by $(5.9), C_{p}^{*}(\theta)$ is obtained in $(5.3)$, and $W_{p, b}^{*}(\theta)$ in $(5.5)$.

Proof. Substituting (5.7) into (4.6) and using (5.8), we obtain

$$
\begin{aligned}
\tilde{q}_{p}(z) & =1-\frac{1-z}{z} \lambda_{p, b} \sum_{m=1}^{\infty} \sum_{j=1}^{m} \int_{0}^{+\infty} P_{p, b}\left(W_{p, b, 0}+C_{p, 0,1}+\cdots+C_{P, 0, m-j}>s\right) z^{j} \\
& \times P_{p, b}\left(X_{p, 0}=m\right) E_{p, b}\left(\tilde{X}_{p}(z) N_{p, b}(0, s)\right) d s \\
& =1-\frac{1-z}{z} \sum_{m=1}^{\infty} P_{p, b}\left(X_{p, 0}=m\right) \sum_{j=1}^{m} z^{j} \frac{1-W_{p, b}^{*}\left(\eta_{p}\right)\left[C_{p}^{*}\left(\eta_{p}\right)\right]^{m-j}}{1-\tilde{X}_{p}(z)} \\
& =(1-z) \frac{\left[\tilde{X}_{p}\left(C_{p}^{*}\left(\eta_{p}\right)\right)-\tilde{X}_{p}(z)\right]}{\left(1-\tilde{X}_{p}(z)\right)\left(C_{p}^{*}\left(\eta_{p}\right)-z\right)} W_{p, b}^{*}\left(\eta_{p}\right)
\end{aligned}
$$

which yields (5.12). Equations (5.10) and (5.11) are seen to be valid if we substitute (5.7) into (4.4) or (4.5), respectively.

\section{Remark 5.2}

a) For a single-class $(I=1)$ case, (5.10) and (5.11) are reduced to Theorem 7.3.7 in Brandt et al. [1], where the service interruptions are not allowed.

b) For a two-class $(I=2)$ non-batch arrival $\left(X_{p} \equiv 1\right) \vec{M} / \overrightarrow{G I} / 1$ (HL) priority queue, equations (5.10) and (5.12) are reduced to the results in Keilson and Servi [11].

c) So far, we have derived the $z$-transform $\tilde{l}_{p}(z)$ for the queue-length distribution in terms of the $\operatorname{LST} W_{p, b}^{*}(\theta)$ for the waiting time distributions. The inverse direction obtaining the $\operatorname{LST} W_{p, b}^{*}(\theta)$ in terms of the $z$-transform $\tilde{l}_{p}(z)$ is possible if $\eta_{p}(z)$ in (5.9) has its inverse function. However, for the $\overrightarrow{G I^{X}} / \overrightarrow{G I / 1}$ (HL or PR) priority queue, the inverse direction is not easy to obtain for lack of the explicit formulas as in Theorem 5.1.

\section{Appendix}

Consider the probability spaces $(\Omega, \mathcal{F}, P)$ and $\left(\Omega, \mathcal{F}, P_{p, b}\right)$. If two events $\mathrm{A}_{1}$ and $\mathrm{A}_{2}$ are independent with respect to $P\left[\right.$ or $\left.P_{p, b}\right]$, they are said to be $P$ [or $\left.P_{p, b}\right]$-independent for brevity. In the following, $A$ is said to be $P\left[\right.$ or $\left.P_{p, b}\right]$-independent of the batch-arrival process $N_{p, b}$, if $A$ is $P$ [or $P_{p, b}$ ]-independent of $B$ for all $B \in \mathcal{F}_{p, b}$ where $\mathcal{F}_{p, b}$ is the sub- $\sigma$-field of $\mathcal{F}$ generated by $N_{p, b}$, i.e.,

$$
\mathcal{F}_{p, b} \equiv \sigma\left\{\left\{N_{p, b}(C)=k\right\}, k \geq 0, C \in \mathcal{B}(\mathcal{R})\right\}
$$

We show the following independence property between $P$ and $P_{p, b}$, which is intuitively trivial, but has not proved yet in the literature.

Lemma A.1 For any $A \in \mathcal{F}$, we have

(a) If $A$ is $P$-independent of $N_{p, b}$, then $A$ is $P_{p, b}$-independent of $N_{p, b}$;

(b) If $A$ is $P_{p, b}$-independent of $N_{p, b}$, and if $\chi_{\mathrm{A}} \circ \mathbf{T}^{s}=\chi_{\mathrm{A}}$ on $\left\{s<T_{p, 1}\right\}\left(a . s . P_{p, b}\right)$, then $A$ is $P$-independent of $N_{p, b}$;

and in these cases,

$$
P(A)=P_{p, b}(A)
$$

Proof of $(a)$ : The $P$-independence and the stationarity imply:

$$
\left.E\left(\chi_{A} \circ \mathrm{T}^{s} \chi_{B}\right)=E\left(\chi_{A} \circ \mathrm{T}^{s}\right) E\left(\chi_{B}\right)=E\left(\chi_{A}\right) E\left(\chi_{B}\right)=E\left(P(A) \chi_{B}\right) \text { (for all } B \in \mathcal{F}_{p, b}\right) \text {. }
$$


Recalling that $P(A)$ is constant and so $\mathcal{F}_{p, b}$-measurable, and remembering the befinition of conditional expectation yield

$$
E\left(\chi_{A} \circ \mathrm{T}^{s} \mid \mathcal{F}_{p, b}\right)=P(A) .
$$

The definition of the Palm distribution (2.1) then gives for all $B \in \mathcal{F}_{p, b}$ :

$$
\begin{aligned}
P_{p, b}(A \cap B) & =\frac{1}{\lambda_{p, b}} E\left(\int_{0}^{1} \chi_{A \cap B} \circ \mathbf{T}^{s} N_{p, b}(d s)\right) \\
& =\frac{1}{\lambda_{p, b}} E\left(E\left(\int_{0}^{1} \chi_{A \cap B} \circ \mathbf{T}^{s} N_{p, b}(d s) \mid \mathcal{F}_{p, b}\right)\right) \\
& =\frac{1}{\lambda_{p, b}} E\left(E\left(\int_{0}^{1} \chi_{A} \circ \mathbf{T}^{s} \chi_{B} \circ \mathbf{T}^{s} N_{p, b}(d s) \mid \mathcal{F}_{p, b}\right)\right) \\
{\left[\text { from } \mathcal{F}_{p, b}\right.} & \left.- \text { measurability of } \chi_{B} \circ \mathbf{T}^{s}\right] \\
& =\frac{1}{\lambda_{p, b}} E\left(\int_{0}^{1} E\left(\chi_{A} \circ \mathbf{T}^{s} \mid \mathcal{F}_{p, b}\right) \chi_{B} \circ \mathbf{T}^{s} N_{p, b}(d s)\right)
\end{aligned}
$$

[by (A.1)]

$$
\begin{aligned}
& =P(A) \frac{1}{\lambda_{p, b}} E\left(\int_{0}^{1} \chi_{B} \circ \mathrm{T}^{s} N_{p, b}(d s)\right) \\
& =P(A) P_{p, b}(B) .
\end{aligned}
$$

Taking $B$ as the total event $(B=\Omega),($ A.1) is reduced to:

$$
P_{p, b}(A)=P(A) \text {. }
$$

Substituting (A.3) into (A.2), we obtain

$$
P_{p, b}(A \cap B)=P_{p, b}(A) P_{p, b}(B),
$$

showing that $A$ and $B$ are $P_{p, b}$-independent for all $B \in \mathcal{F}_{p, b}$.

Proof of $(b)$ : Note that $P_{p, b}$-independence and the additional assumption for $\chi_{A} \circ \mathrm{T}^{s}=\chi_{A}$ on $\left\{s<T_{p, 1}\right\}$ imply:

$$
\begin{aligned}
E_{p, b}\left(\chi_{A} \circ \mathrm{T}^{s} \chi_{\left\{T_{p, 1}>s\right\}} \chi_{B}\right) & =E_{p, b}\left(\chi_{A} \chi_{\left\{T_{p, 1}>s\right\}} \chi_{B}\right)=P_{p, b}(A) E_{p, b}\left(\chi_{\left\{T_{p, 1}>s\right\}} \chi_{B}\right) \\
& =E_{p, b}\left(P_{p, b}(A) \chi_{\left\{T_{p, 1}>s\right\}} \chi_{B}\right) \quad\left(\text { for all } B \in \mathcal{F}_{p, b}\right) .
\end{aligned}
$$

Recalling that $P_{p, b}(A) \chi_{\left\{T_{p, 1}>s\right\}}$ is $\mathcal{F}_{p, b^{-}}$measurable, and remembering the definition of conditional expectation yield

$$
E_{p, b}\left(\chi_{A} \circ \mathrm{T}^{s} \mid \mathcal{F}_{p, b}\right) \chi_{\left\{T_{p, 1}>s\right\}}=P_{p, b}(A) \chi_{\left\{T_{p, 1}>s\right\}} .
$$

The inversion formula (2.3) then gives for all $B \in \mathcal{F}_{p, b}$ :

[by (A.4)]

$$
\begin{aligned}
P(A \cap B) & =\lambda_{p, b} E_{p, b}\left(\int_{0}^{T_{p, 1}} \chi_{A \cap B} \circ \mathbf{T}^{s} d s\right) \\
& =\lambda_{p, b} E_{p, b}\left(\int_{0}^{\infty} \chi_{\left\{T_{p, 1}>s\right\}} \chi_{A \cap B} \circ \mathbf{T}^{s} d s\right) \\
& =\lambda_{p, b} E_{p, b}\left(E_{p, b}\left(\int_{0}^{\infty} \chi_{\left\{T_{p, 1}>s\right\}} \chi_{A \cap B} \circ \mathbf{T}^{s} d s \mid \mathcal{F}_{p, b}\right)\right) \\
& =\lambda_{p, b} E_{p, b}\left(\int_{0}^{\infty} E_{p, b}\left(\chi_{A} \circ \mathbf{T}^{s} \mid \mathcal{F}_{p, b}\right) \chi_{\left\{T_{p, 1}>s\right\}} \chi_{B} \circ \mathbf{T}^{s} d s\right)
\end{aligned}
$$

$$
\begin{aligned}
& =P_{p, b}(A) \lambda_{p, b} E_{p, b}\left(\int_{0}^{\infty} \chi_{\left\{T_{p, 1}>s\right\}} \chi_{B} \circ \mathbf{T}^{s} d s\right) \\
& =P_{p, b}(A) P(B)
\end{aligned}
$$


Taking $B$ as the total event $\Omega(B=\Omega),($ A.5) is reduced to (A.3). Substituting (A.3) into (A.5), we obtain

$$
P(A \cap B)=P(A) P(B),
$$

which shows that $A$ and $B$ are $P$-independent for all $B \in \mathcal{F}_{p, b}$.

It is clear that Lemma A.1 also holds for $P$ and $P_{p, c}$, if we replace index $(p, b)$ by $(p, c)$.

\section{Acknowledgements}

The authors wish to thank the first referee for his critical and constructive comments.

\section{References}

[1] A. Brandt, P. Franken and B. Lisek, Stationary Stochastic Models (John Wiley, New York, 1990).

[2] S. L. Brumelle, A generalization of $L=\lambda W$ to moments of queue length and waiting times, Operations Research, 20, 1127-1136 (1972).

[3] P. J. Burke, Delays in single-server queues with batch input, Operations Research, 23, 830-833 (1975).

[4] M. L. Chaudhry and J. G. C. Templeton, A First Course in Bulk Queues (John Wiley, New York, 1983).

[5] R. B. Cooper, Introduction to Queueing Theory (North-Holland, New York, 1981).

[6] P. Franken, D. König, U. Arndt and V. Schmidt, Queues and Point Processes (John Wiley, Chichester, 1982).

[7] R. Haji and G. F. Newell, A relation between stationary queue and waiting distributions, J. Appl. Prob., 8, 617-620 (1971).

[8] A. G. Hawkes, Time dependent solution of a priority queue with bulk arrival, Operations Research, 13, 586-595 (1965).

[9] N. K. Jaiswal, Priority Queues (Academic Press, New York, 1968).

[10] J. Keilson and L. D. Servi, A distributional form of Little's law, Operations Research Letters, 7, 223-227 (1988).

[11] J. Keilson and L. D. Servi, The distributional form of Little's law and the FuhrmannCooper decomposition, Operations Research Letters, 9, 239-247 (1990).

[12] O. Kella and U. Yechiali, Priorities in $M / G / 1$ queue with server vacations, Naval Research Logistics, 35, 23-34 (1988).

[13] D. König, T. Rolski, V. Schmidt and D. Stoyan, Stochastic processes with imbedded marked point processes (PMP) and their applications in queueing, Math. Operationsforsch. Statist., 9, 125-141 (1978).

[14] B. Meister, Waiting time in a preemptive resume system with compound Poisson input, Computing, 25, 17-28 (1980).

[15] M. Miyazawa, Time and customer processes in queues with stationary inputs, J. Appl. Prob., 14, 349-357 (1977).

[16] M. Miyazawa, A formal approach to queueing processes in the steady state and their applications, J. Appl. Prob., 16, 332-346 (1979).

[17] M. Miyazawa, Comparison of the loss probability of the $G I^{X} / G I / k$ queues with a common traffic intensity, Journal of the Operations Research Society of Japan, 32, 505-516 (1989).

[18] H. Takagi and Y. Takahashi, Priority queues with batch Poisson arrivals, Operations Research Letters, 10, 225-232 (1991).

[19] Y. Takahashi, On the relationship between work load and waiting time in single server queues with batch inputs, Operations Research Letters, 7, 51-56 (1988). 
[20] Y. Takahashi and H. Takagi, Structured priority queue with batch arrivals, Journal of the Operations Research Society of Japan, 33, 242-261 (1990).

[21] Y. Takahashi and S. Shimogawa, Composite priority single-server queue with structured batch inputs Stochastic Models, 7, 481-497 (1991).

[22] W. Whitt, A review of $L=\lambda W$ and extensions, Queueing Systems, 9, (1991) 235-268.

[23] R. W. Wolff, Stochastic Modeling and the Theory of Queues (Prentice Hall, New Jersey, 1989).

Yoshitaka TAKAHASHI

Performance Evaluation Research Group

Network Traffic Laboratory

NTT Telecommunication Networks Laboratories

Musashino-shi, Tokyo 180, JAPAN

Masakiyo MIYAZAWA

Department of Information Sciences

Faculty of Science and Technology

Science University of Tokyo

Noda-shi, Chiba 278, JAPAN

Under $P_{p, b}$ measure
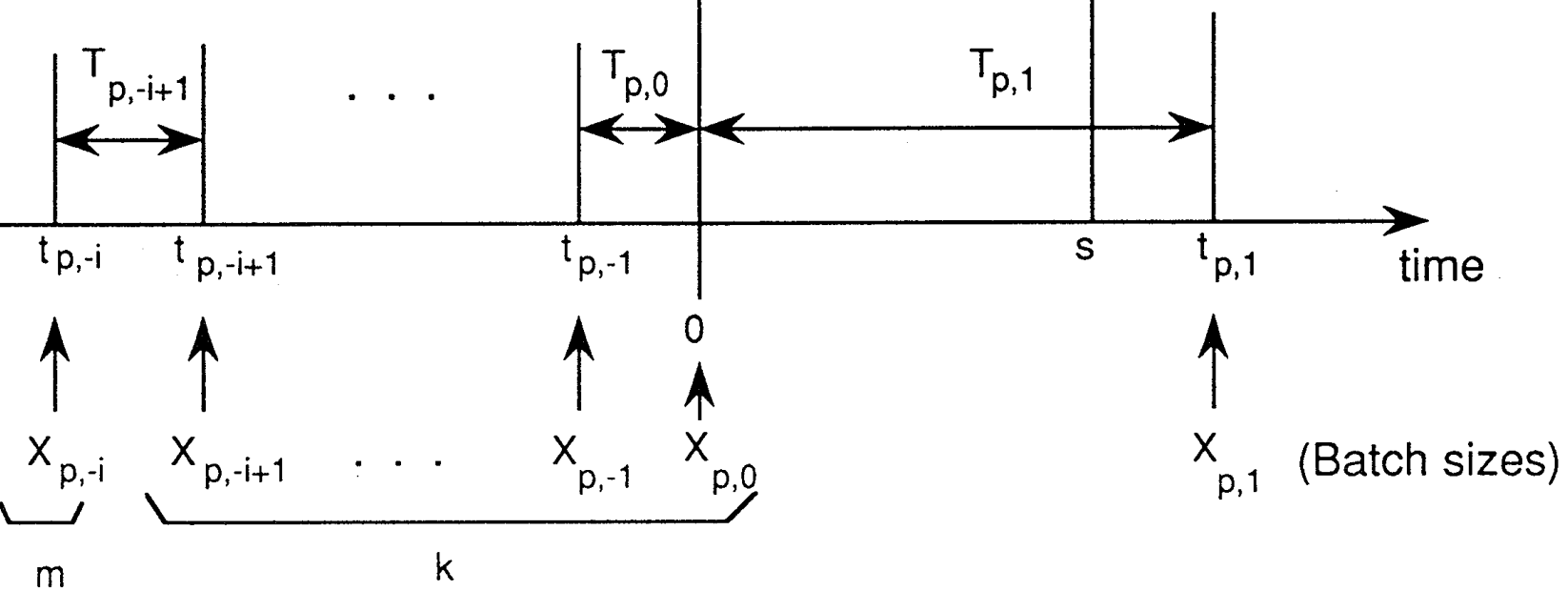

The first customer of $X_{p,-i}$ still remains in the system

$\Leftrightarrow \quad W_{p, b,-i}+S_{p,-i, 1}>T_{p,-i+1}+\ldots+T_{p, 0}+s$

Figure 1: Key observation for the single-server priority queue with batch arrivals. 